\title{
Atmospheric characterization of the ultra-hot Jupiter MASCARA-2b/KELT-20b
}

\section{Detection of Ca II, Fe II, Na I, and the Balmer series of $\mathrm{H}(\mathrm{H} \alpha, \mathrm{H} \beta$, and $\mathrm{H} \gamma)$ with high-dispersion transit spectroscopy (Corrigendum)}

\author{
N. Casasayas-Barris ${ }^{1,2}$, E. Pallé ${ }^{1,2}$, F. Yan $^{3}$, G. Chen ${ }^{1,2,4}$, S. Kohl ${ }^{5}$, M. Stangret ${ }^{1,2}$, H. Parviainen ${ }^{1,2}$, \\ Ch. Helling ${ }^{6,7,8}$, N. Watanabe ${ }^{9,10}$, S. Czesla ${ }^{5}$, A. Fukui ${ }^{14,1}$, P. Montañés-Rodríguez ${ }^{1,2}$, E. Nagel ${ }^{5}$, N. Narita ${ }^{9,11,12,1}$, \\ L. Nortmann ${ }^{1,2}$, G. Nowak ${ }^{1,2}$, J. H. M. M. Schmitt ${ }^{5}$, and M. R. Zapatero Osorio ${ }^{13}$ \\ ${ }^{1}$ Instituto de Astrofísica de Canarias, Vía Láctea s/n, 38205 La Laguna, Tenerife, Spain \\ e-mail: nuriacb@iac.es \\ 2 Departamento de Astrofísica, Universidad de La Laguna, La Laguna, Spain \\ ${ }^{3}$ Institut für Astrophysik, Georg-August-Universität, Friedrich- Hund-Platz 1, 37077 Göttingen, Germany \\ ${ }^{4}$ Key Laboratory of Planetary Sciences, Purple Mountain Observatory, Chinese Academy of Sciences, Nanjing 210008, PR China \\ ${ }^{5}$ Hamburger Sternwarte, Universität Hamburg, Gojenbergsweg 112, 21029 Hamburg, Germany \\ ${ }^{6}$ Centre for Exoplanet Science, University of St Andrews, St Andrews, UK \\ 7 SUPA, School of Physics \& Astronomy, University of St Andrews, St Andrews, KY16 9SS, UK \\ ${ }^{8}$ SRON Netherlands Institute for Space Research, Sorbonnelaan 2, 3584 CA Utrecht, The Netherlands \\ ${ }^{9}$ National Astronomical Observatory of Japan, 2-21-1 Osawa, Mitaka, Tokyo 181-8588, Japan \\ ${ }^{10}$ SOKENDAI (The Graduate University of Advanced Studies), 2-21-1 Osawa, Mitaka, Tokyo 181-8588, Japan \\ ${ }_{11}$ Astrobiology Center, 2-21-1 Osawa, Mitaka, Tokyo 181-8588, Japan \\ 12 JST, PRESTO, 2-21-1 Osawa, Mitaka, Tokyo 181-8588, Japan \\ ${ }^{13}$ Centro de Astrobiología (CSIC-INTA), Carretera de Ajalvir km 4, 28850 Torrejón de Ardoz, Madrid, Spain \\ ${ }^{14}$ Department of Earth and Planetary Science, Graduate School of Science, The University of Tokyo, 7-3-1 Hongo, Bunkyo-ku, Tokyo \\ 113-0033, Japan
}

A\&A, 628, A9 (2019), https://doi .org/10.1051/0004-6361/201935623

Key words. planetary systems - methods: observational - techniques: spectroscopic planets and satellites: individual: MASCARA-2b - planets and satellites: individual: KELT-20b - errata, addenda

The original article has two errors which are corrected below and which do not have any impact on the conclusions.

1. In Fig. 7 of the original article, the labels inside the plot are inverted. The corrected figure is shown in Fig. 1.

2. There was an error in the constant value used for the conversion between the standard deviation $(\sigma)$ and the full width at half maximum (FWHM) when fitting the exoplanet atmospheric absorption with a Gaussian profile. As a result, all of the FWHM values shown in the original article change by a constant value. The affected tables and figures from the main text are those containing FWHM information, that is to say, Tables 4, 5, and Fig. 13. In the appendix, this affects Table B.1 and all corner plots from Appendix D (Figs. D.1D.7). The corrected tables and figures are presented below in the same order as in the original article.

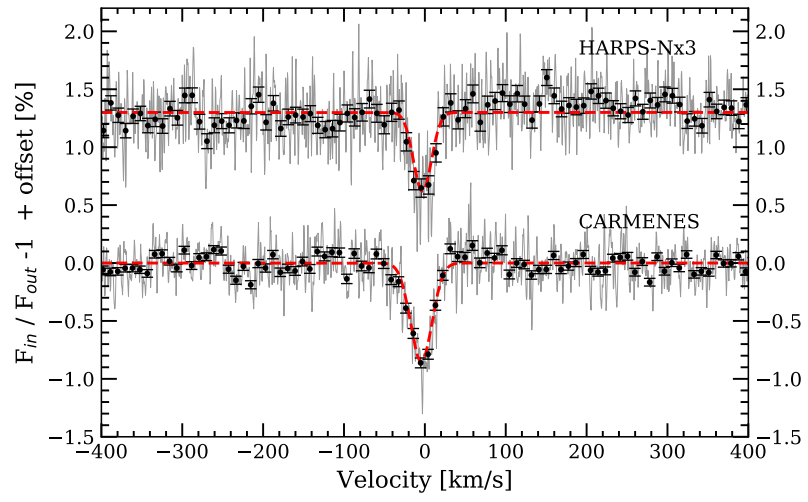

Fig. 1. Transmission spectra around $\mathrm{H} \alpha$ line obtained with all nights of HARPS-N combined and CARMENES data (Night 4), with an offset for a better visualisation. The best-fit Gaussian profiles are shown in red. In grey, we show the original transmission spectrum and the black dots show the data binned in $0.2 \AA$ intervals. 
Table 1. Best fit parameters and 1- $\sigma$ error bars from the Markov chain Monte Carlo (MCMC) analysis of the H $\alpha$ line for each individual night and combined HARPS-N observations.

\begin{tabular}{lcccccc}
\hline \hline & $\begin{array}{c}h \\
{[\%]}\end{array}$ & $\begin{array}{c}F W H M \\
{\left[\mathrm{~km} \mathrm{~s}^{-1}\right]}\end{array}$ & $\begin{array}{c}K_{\mathrm{p}} \\
{\left[\mathrm{km} \mathrm{s}^{-1}\right]}\end{array}$ & $\begin{array}{c}v_{\text {wind }} \\
{\left[\mathrm{km} \mathrm{s}^{-1}\right]}\end{array}$ & $\begin{array}{c}R_{\lambda}{ }^{(*)} \\
{\left[R_{\mathrm{p}}\right]}\end{array}$ & $\begin{array}{c}R_{\lambda}(h)(\S) \\
{\left[R_{\mathrm{p}}\right]}\end{array}$ \\
\hline HARPS-N 1 & $-0.75_{-0.11}^{+0.10}$ & $48.4_{-8.9}^{+10.7}$ & $140.3_{-42.1}^{+39.0}$ & $1.3_{-2.9}^{+3.0}$ & $1.21_{-0.05}^{+0.04}$ & $1.24_{-0.06}^{+0.06}$ \\
\hline HARPS-N 2 & $-0.52_{-0.11}^{+0.10}$ & $21.6_{-5.4}^{+5.1}$ & $101.5_{-33.5}^{+33.7}$ & $-3.9_{-2.4}^{+2.1}$ & $1.08_{-0.03}^{+0.03}$ & $1.17_{-0.07}^{+0.06}$ \\
\hline HARPS-N 3 & $-0.69_{-0.11}^{+0.10}$ & $22.7_{-3.3}^{+3.6}$ & $196.0_{-32.0}^{+30.1}$ & $-2.8_{-2.2}^{+2.2}$ & $1.15_{-0.04}^{+0.04}$ & $1.22_{-0.06}^{+0.06}$ \\
\hline CARMENES 1 & $-0.85_{-0.03}^{+0.03}$ & $31.3_{-1.2}^{+1.3}$ & $166.2_{-7.4}^{+7.3}$ & $-4.5_{-0.5}^{+0.5}$ & $1.21_{-0.01}^{+0.01}$ & $1.27_{-0.02}^{+0.02}$ \\
\hline HARPS-Nx3 & $-0.68_{-0.06}^{+0.06}$ & $26.3_{-2.2}^{+2.4}$ & $165.6_{-16.6}^{+16.7}$ & $-3.0_{-1.2}^{+1.2}$ & $1.13_{-0.02}^{+0.02}$ & $1.22_{-0.04}^{+0.04}$ \\
\hline
\end{tabular}

Notes. ${ }^{(*)}$ Effective radius value obtained from the best fit model of the CLV and RME effects. ${ }^{(\S)}$ Effective radius was calculated considering the absorption value, $h$, and assuming a continuum level of $\left(R_{\mathrm{p}} / R_{\star}\right)^{2}=1.382 \%$.

Table 2. Summary of the results obtained in the analysis is presented here for the different species and instruments.

\begin{tabular}{lccccccccc}
\hline \hline & & $h[\%]$ & $R_{\lambda}(h)\left[R_{\mathrm{p}}\right]$ & $v_{\text {wind }}\left[\mathrm{km} \mathrm{s}^{-1}\right]$ & $F W H M\left[\mathrm{~km} \mathrm{~s}^{-1}\right]$ & $K_{\mathrm{p}}\left[\mathrm{km} \mathrm{s}^{-1}\right]$ & $\mathrm{AD}_{\mathrm{TS}}^{1.5 \AA}[\%]^{(*)}$ & $\mathrm{AD}_{\mathrm{TLC}}^{1.5 \AA}[\%]^{(\ddagger)}$ & $\mathrm{AD}_{\mathrm{EMC}}^{1.5 \AA}[\%]^{(\mathbb{I l})}$ \\
\hline $\mathrm{H} \alpha$ & $\mathrm{Hx} 3$ & $-0.68 \pm 0.06$ & $1.22 \pm 0.04$ & $-3.0 \pm 1.2$ & $26.3 \pm 2.4$ & $165.6 \pm 16.7$ & $0.33 \pm 0.05$ & $0.30 \pm 0.05$ & $0.36 \pm 0.14$ \\
& $\mathrm{Cx} 1$ & $-0.85 \pm 0.03$ & $1.27 \pm 0.02$ & $-4.5 \pm 0.5$ & $31.3 \pm 1.3$ & $166.2 \pm 7.4$ & $0.44 \pm 0.04$ & $0.43 \pm 0.05$ & $0.40 \pm 0.14$ \\
\hline $\mathrm{H} \beta$ & $\mathrm{Hx} 3$ & $-0.45 \pm 0.05$ & $1.15 \pm 0.03$ & $-1.2 \pm 1.4$ & $26.9 \pm 3.5$ & $136.2 \pm 18.6$ & $0.13 \pm 0.04$ & $0.17 \pm 0.04$ & $0.14 \pm 0.08$ \\
\hline $\mathrm{H} \gamma$ & $\mathrm{Hx} 3$ & $-0.38 \pm 0.08$ & $1.13 \pm 0.05$ & $-2.3 \pm 2.7$ & $23.0 \pm 5.8$ & $135.0 \pm 34.8$ & $0.09 \pm 0.05$ & $0.07 \pm 0.09$ & $0.08 \pm 0.04$ \\
\hline $\mathrm{CaII}$ & $\mathrm{Cx} 1$ & $-0.56 \pm 0.05$ & $1.18 \pm 0.03$ & $-1.9 \pm 0.6$ & $12.7 \pm 1.4$ & $157.7 \pm 8.2$ & $0.16 \pm 0.04$ & $0.14 \pm 0.04$ & $0.11 \pm 0.09$ \\
\hline \multirow{2}{*}{$\mathrm{NaI}$} & $\mathrm{Hx} 3$ & $-0.34 \pm 0.05$ & $1.11 \pm 0.03$ & $-3.1 \pm 0.9$ & $12.7 \pm 2.8$ & $182.5 \pm 14.3$ & $0.05 \pm 0.03$ & $0.08 \pm 0.03$ & $0.07 \pm 0.05$ \\
& $\mathrm{Cx} 1$ & $-0.29 \pm 0.04$ & $1.10 \pm 0.03$ & $-3.2 \pm 0.7$ & $11.1 \pm 1.7$ & $176.6 \pm 11.7$ & $0.04 \pm 0.03$ & $0.03 \pm 0.04$ & $0.08 \pm 0.08$ \\
\hline $\mathrm{FeII}$ & $\mathrm{Hx} 3$ & $-0.33 \pm 0.05$ & $1.11 \pm 0.03$ & $-2.8 \pm 0.8$ & $10.0 \pm 1.7$ & $174.4 \pm 14.0$ & $0.04 \pm 0.03$ & $0.01 \pm 0.03$ & $0.04 \pm 0.04$ \\
\hline
\end{tabular}

Notes. Hx 3 corresponds to the analysis of three HARPS-N nights combined and Cx1 refers to the analysis of the unique transit observed with CARMENES. ${ }^{(*)}$ Absorption depth measured in the transmission spectrum for a $1.5 \AA$ passband. ${ }^{(\ddagger)}$ Absorption depth measured in the transmission light curve for a $1.5 \AA$ passband. ${ }^{(\mathbb{I I})}$ Centre of the EMC distribution was computed for a $1.5 \AA$ passband.

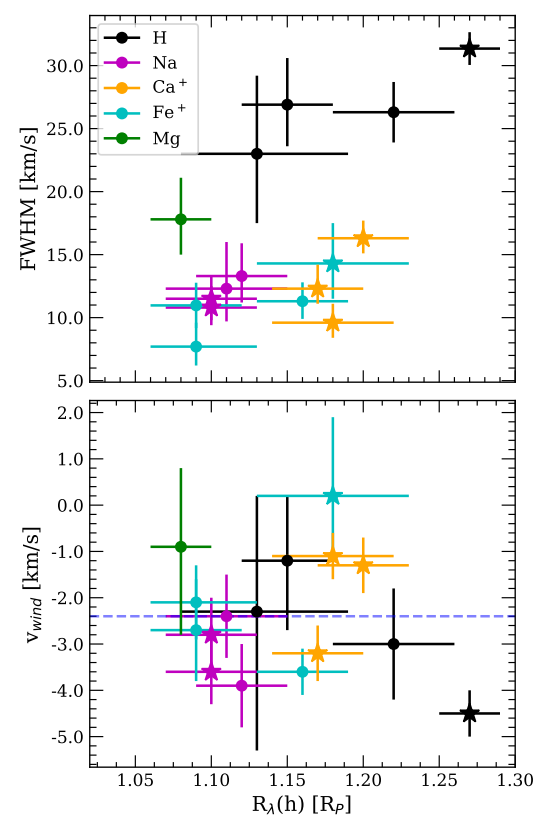

Fig. 2. Top: FWHM values vs. $R_{\lambda}(h)$. Bottom: $v_{\text {wind }}$ values vs. $R_{\lambda}(h)$. The measurements obtained with HARPS-N data are shown with dots, while those obtained with CARMENES data are marked with stars. The horizontal blue dashed line shows the mean $v_{\text {wind }}$ value at $-2.4 \pm 1.0 \mathrm{~km} \mathrm{~s}^{-1}$. All values were extracted from Table 2 . 
Table 3. Best fit parameters and 1- $\sigma$ error bars from the MCMC analysis.

\begin{tabular}{|c|c|c|c|c|c|c|c|}
\hline & & $\begin{array}{c}h \\
{[\%]}\end{array}$ & $\begin{array}{l}F W H M \\
{\left[\mathrm{~km} \mathrm{~s}^{-1}\right]}\end{array}$ & $\begin{array}{c}K_{\mathrm{p}} \\
{\left[\mathrm{km} \mathrm{s}^{-1}\right]}\end{array}$ & $\begin{array}{c}v_{\text {wind }} \\
{\left[\mathrm{km} \mathrm{s}^{-1}\right]}\end{array}$ & $\begin{array}{c}R_{\lambda}{ }^{* *} \\
{\left[R_{\mathrm{p}}\right]}\end{array}$ & $\begin{array}{c}R_{\lambda}(h)^{(\S)} \\
{\left[R_{\mathrm{p}}\right]} \\
\end{array}$ \\
\hline \multirow[t]{4}{*}{$\overline{\mathrm{H} \beta}$} & HARPS-N 1 & $-0.20_{-0.19}^{+0.16}$ & $20.4_{-13.0}^{+22.2}$ & $110.6_{-71.7}^{+85.5}$ & $-5.1_{-6.6}^{+8.0}$ & $1.12_{-0.03}^{+0.04}$ & $1.07_{-0.13}^{+0.11}$ \\
\hline & HARPS-N 2 & $-0.57_{-0.08}^{+0.07}$ & $22.8_{-3.6}^{+3.9}$ & $131.8_{-18.4}^{+19.3}$ & $-1.5_{-1.7}^{+1.6}$ & $0.89_{-0.03}^{+0.03}$ & $1.19_{-0.05}^{+0.04}$ \\
\hline & HARPS-N 3 & $-0.38_{-0.09}^{+0.08}$ & $33.5_{-8.3}^{+9.7}$ & $166.3_{-43.7}^{+55.7}$ & $+4.2_{-3.5}^{+3.6}$ & $0.86_{-0.03}^{+0.03}$ & $1.13_{-0.06}^{+0.05}$ \\
\hline & HARPS-Nx3 & $-0.45_{-0.05}^{+0.05}$ & $26.9_{-3.3}^{+3.7}$ & $136.2_{-18.5}^{+18.7}$ & $-1.2_{-1.5}^{+1.4}$ & $0.91_{-0.02}^{+0.02}$ & $1.15_{-0.03}^{+0.03}$ \\
\hline \multirow[t]{4}{*}{$\mathrm{H} \gamma$} & HARPS-N 1 & $-0.29_{-0.26}^{+0.13}$ & $40.9_{-21.9}^{+78.0}$ & $189.7_{-105.8}^{+43.7}$ & $-1.4_{-6.3}^{+7.2}$ & $1.01_{-0.05}^{+0.05}$ & $1.10_{-0.17}^{+0.08}$ \\
\hline & HARPS-N 2 & $-0.43_{-0.12}^{+0.10}$ & $23.4_{-6.4}^{+7.8}$ & $101.5_{-105.8}^{+43.7^{\circ}}$ & $-4.0_{-30}^{+2.8}$ & $0.86_{-0.03}^{+0.03}$ & $1.15_{-0.08}^{+0.07}$ \\
\hline & HARPS-N 3 & $-0.28_{-0.23}^{+0.17}$ & $25.6_{-15 .}^{+25.8}$ & $104.6_{-65.8}^{+81.0}$ & $-2.8_{-7.5}^{+8.5}$ & $0.78_{-0.05}^{+0.05}$ & $1.13_{-0.06}^{+0.05}$ \\
\hline & HARPS-Nx3 & $-0.38_{-0.09}^{+0.08}$ & $23.0_{-5.5}^{+6.2}$ & $135.0_{-35.6}^{+34.1}$ & $\begin{array}{r}-2.3_{-3.0}^{+2.5} \\
\end{array}$ & $0.85_{-0.03}^{+0.02}$ & $1.13_{-0.06}^{+0.05}$ \\
\hline \multirow[t]{5}{*}{$\mathrm{NaI} \mathrm{D}_{2}$} & HARPS-N 1 & $-0.37_{-0.16}^{+0.14}$ & $8.2_{-3.0}^{+5.5}$ & $151.4_{-36.4}^{+35.4}$ & $-0.9_{-2.6}^{+2.5}$ & $0.92_{-0.05}^{+0.05}$ & $1.13_{-0.10}^{+0.09}$ \\
\hline & HARPS-N 2 & $-0.12_{-0.02}^{+0.02}$ & $133.6_{-8.3}^{+3.7}$ & $137.1_{-91.3}^{+78.3}$ & $1.4_{-96}^{+9.0}$ & $0.97_{-0.04}^{+0.04}$ & $1.04_{-0.01}^{+0.01}$ \\
\hline & HARPS-N 3 & $-0.28_{-0.10}^{+0.02}$ & $20.6_{-86}^{+8.3}$ & $103.3_{-264}^{+26.1}$ & $-1.3_{-2.4}^{+2.6}$ & $0.94_{-0.04}^{-0.04}$ & $1.10_{-0.07}^{+0.01}$ \\
\hline & HARPS-Nx3 & $-0.33_{-0.06}^{+0.06}$ & $12.3_{-2.7}^{+3.7}$ & $171.1_{-12.8}^{+15.2}$ & $-2.4_{-0.9}^{+0.9}$ & $0.94_{-0.03}^{+0.03}$ & $1.11_{-0.04}^{+0.04}$ \\
\hline & CARMENES 1 & $-0.29_{-0.04}^{+0.00}$ & $11.6_{-1.6}^{+1.8}$ & $176.5_{-12.3}^{+12.8}$ & $-2.8_{-0.8}^{+0.9}$ & $0.91_{-0.02}^{+0.03}$ & $1.10_{-0.03}^{+0.03}$ \\
\hline \multirow[t]{5}{*}{$\mathrm{NaI} \mathrm{D}_{1}$} & HARPS-N 1 & $-0.29_{-0.11}^{+0.11}$ & $14.1_{-4.4}^{+7.1}$ & $177.8_{-41.4}^{+39.9}$ & $-2.2_{-2.4}^{+2.4}$ & $0.97_{-0.05}^{+0.06}$ & $1.10_{-0.07}^{+0.07}$ \\
\hline & HARPS-N 2 & $-0.12_{-0.02}^{+0.01}$ & $132.8_{-10.4}^{+4.4}$ & $143.3_{-93.1}^{+75.4}$ & $-7.4_{-5.7}^{+8.6}$ & $0.90_{-0.03}^{+0.04}$ & $1.04_{-0.01}^{+0.01}$ \\
\hline & HARPS-N 3 & $-0.09_{-0.02}^{+0.02}$ & $113.3_{-302}^{+17.9}$ & $196.5_{-793}^{+40.9}$ & $-6.4_{-6.1}^{+9.6}$ & $0.97_{-0.04}^{+0.04}$ & $1.03_{-0.02}^{+0.01}$ \\
\hline & HARPS-Nx3 & $-0.35_{-0.05}^{+0.02}$ & $13.3_{-2.1}^{+2.6 .2}$ & $194.0_{-14.4}^{+15.3}$ & $\begin{array}{r}-0.1 \\
-3.9_{-0.9}^{+0.9}\end{array}$ & $0.91_{-0.03}^{+0.04}$ & $1.12_{-0.03}^{+0.02}$ \\
\hline & CARMENES 1 & $-0.29_{-0.04}^{+0.04}$ & $10.9_{-1.4}^{+1.7}$ & $176.8_{-10.6}^{+10.8}$ & $-3.6_{-0.7}^{+0.7}$ & $0.90_{-0.02}^{+0.02}$ & $1.10_{-0.03}^{+0.03}$ \\
\hline CaII $\lambda 8498 \AA$ & CARMENES 1 & $-0.52_{-0.05}^{+0.05}$ & $12.3_{-1.2}^{+1.9}$ & $173.2_{-7.5}^{+7.8}$ & $-3.2_{-0.6}^{+0.6}$ & $0.87_{-0.03}^{+0.03}$ & $1.17_{-0.03}^{+0.03}$ \\
\hline CaII $\lambda 8542 \AA$ & CARMENES 1 & $-0.60_{-0.04}^{+0.04}$ & $16.3_{-1.3}^{+1.4}$ & $139.2_{-9.2}^{+8.9}$ & $-1.3_{-0.6}^{+0.6}$ & $1.09_{-0.02}^{+0.02}$ & $1.20_{-0.03}^{+0.03}$ \\
\hline CaII $\lambda 8662 \AA$ & CARMENES 1 & $\begin{array}{r}-0.04 \\
-0.55_{-0.06}^{+0.06} \\
\end{array}$ & $\begin{array}{c}9.6_{-1.3}^{+1.5} \\
\end{array}$ & $160.7_{-7.8}^{+8.2}$ & $-1.1_{-0.5}^{+0.0}$ & $0.95_{-0.03}^{+0.02}$ & $1.18_{-0.04}^{+0.04}$ \\
\hline FeII $\lambda 5018 \AA$ & HARPS-Nx3 & $-0.26_{-0.05}^{+0.04}$ & $11.0_{-1.8}^{+1.8}$ & $181.9_{-18.7}^{+18.3}$ & $-2.7_{-1.1}^{+1.1}$ & $1.19_{-0.03}^{+0.03}$ & $1.09_{-0.03}^{+0.03}$ \\
\hline FeII $\lambda 5169 \AA$ & HARPS-Nx3 & $-0.47_{-0.05}^{+0.05}$ & $11.3_{-1.4}^{+1.8}$ & $170.6_{-7.8}^{+8.3}$ & $-3.6_{-0.5}^{+-0.1}$ & $1.18_{-0.02}^{+0.03}$ & $1.16_{-0.03}^{+0.03}$ \\
\hline \multirow[t]{2}{*}{ FeII $\lambda 5316 \AA$} & HARPS-Nx3 & $-0.26_{-0.06}^{-0.05}$ & $7.8_{-1.5}^{+2.4}$ & $170.8_{-16.6}^{+14.4}$ & $-2.1_{-0.8}^{+0.3}$ & $1.18_{-0.03}^{+0.03}$ & $1.09_{-0.04}^{+0.03}$ \\
\hline & CARMENES 1 & $-0.55_{-0.09}^{+0.08}$ & $14.3_{-2.8}^{+3.2}$ & $139.6_{-22.6}^{+32.3}$ & $+0.2_{-1.3}^{+1.7}$ & $1.24_{-0.06}^{+0.05}$ & $1.18_{-0.05}^{+0.05}$ \\
\hline $\operatorname{MgI} \lambda 5173 \AA$ & HARPS-Nx3 & $-0.23_{-0.04}^{+0.03}$ & $17.8_{-2.7}^{+3.4}$ & $159.6_{-23.8}^{+30.9}$ & $-0.9_{-1.9}^{+1.7}$ & $1.23_{-0.02}^{+0.02}$ & $1.08_{-0.02}^{+0.02}$ \\
\hline
\end{tabular}

Notes. ${ }^{(*)}$ Effective radius value obtained from the best fit model of the CLV and RME effects. ${ }^{(\S)}$ Effective radius was calculated considering the absorption value, $h$, from the best-fit model and assuming a continuum level of $\left(R_{\mathrm{p}} / R_{\star}\right)^{2}=1.382 \%$.
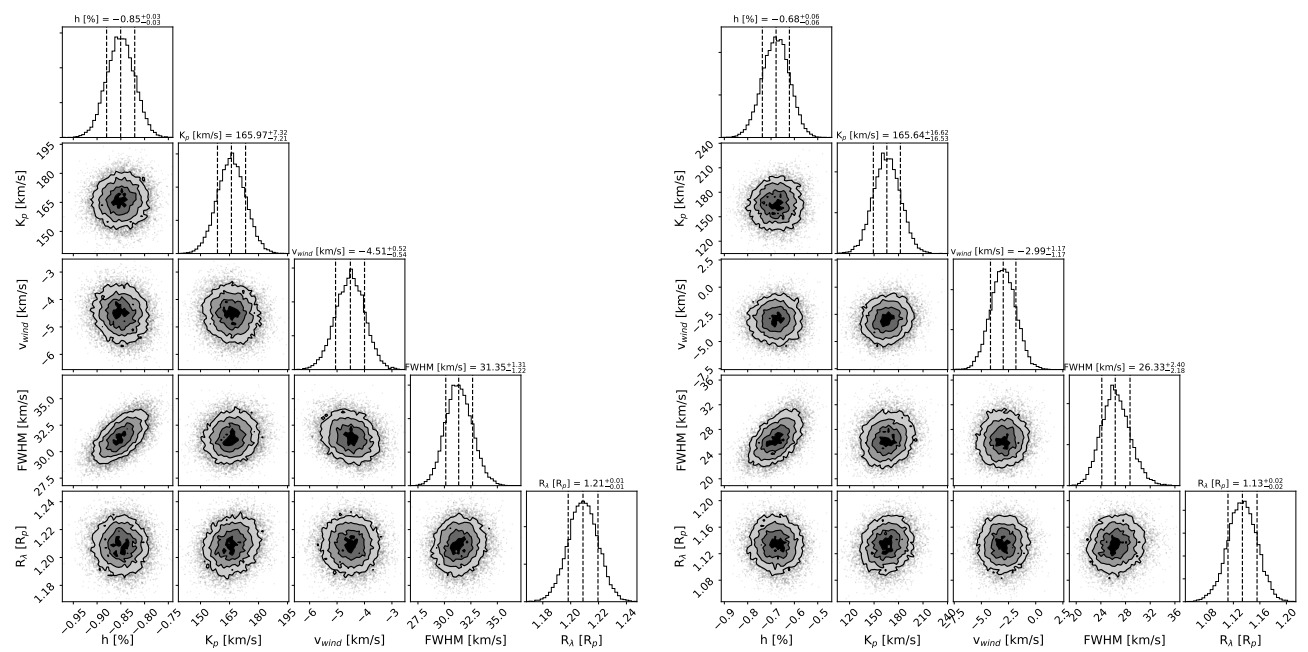

Fig. 3. Correlation diagrams of MCMC analysis of the H $\alpha$ line of CARMENES (left) and HARPS-N data analysis (right). 
A\&A 640, C6 (2020)
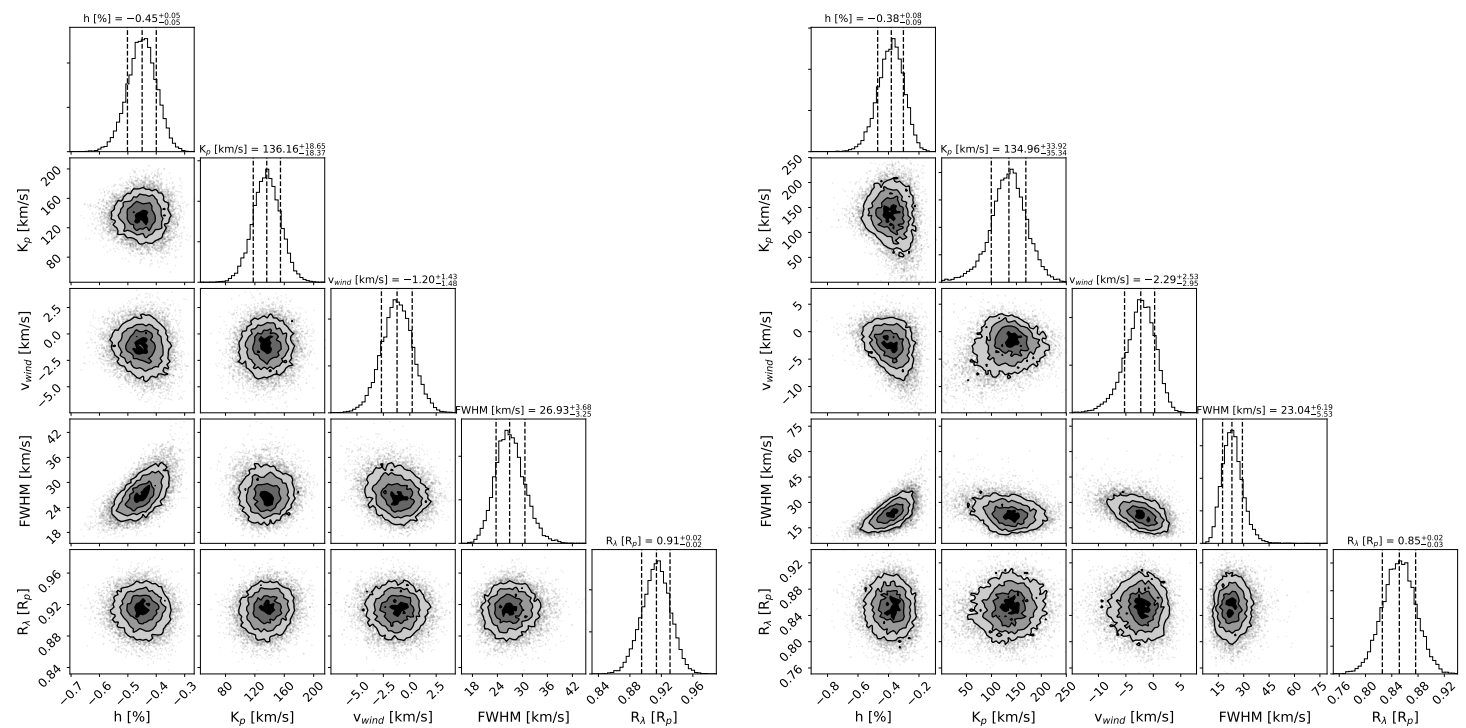

Fig. 4. Correlation diagrams of MCMC analysis of the $\mathrm{H} \beta$ (left) and $\mathrm{H} \gamma$ (right) lines obtained with the HARPS-N data analysis.
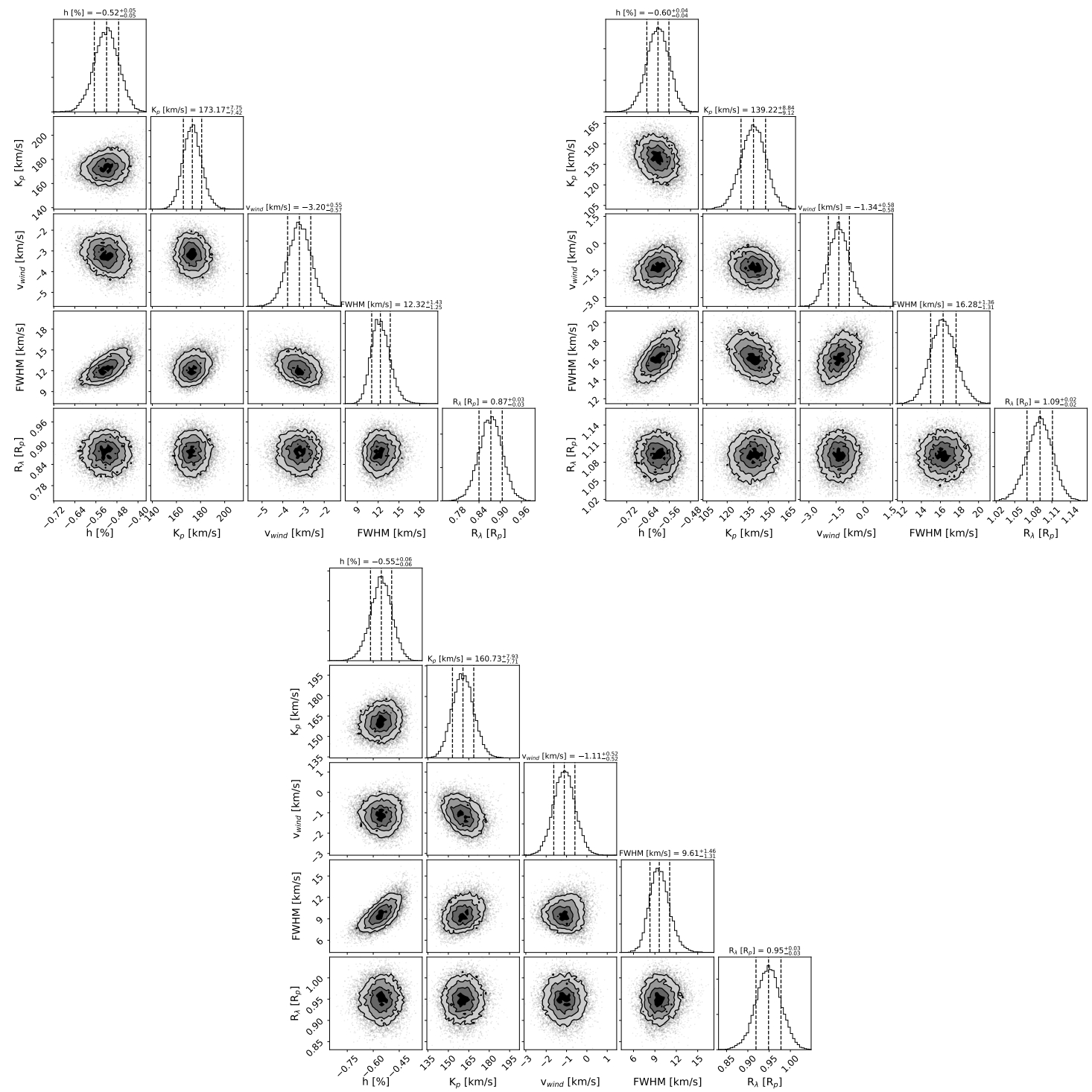

Fig. 5. Correlation diagrams of MCMC analysis of the Ca II triplet lines of CARMENES. The Ca II $\lambda 8498 \AA$ A diagram is shown in the top left panel, Ca II $\lambda 8542 \AA$ is in the top right panel, and $\lambda 8662 \AA$ is in the bottom panel. 
N. Casasayas-Barris et al.: Atmospheric characterization of the ultra-hot Jupiter MASCARA-2b/KELT-20b
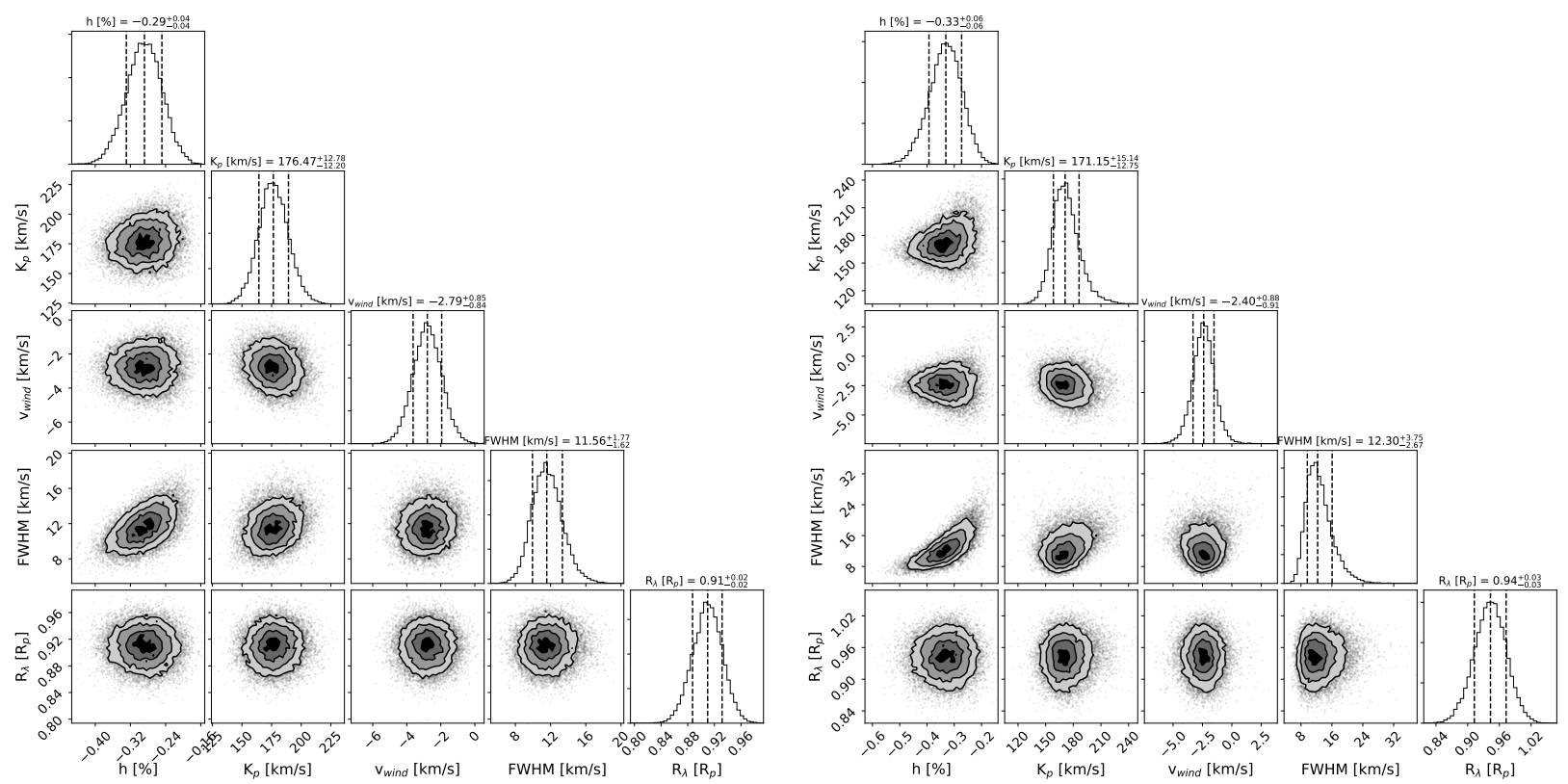

Fig. 6. Correlation diagrams of MCMC analysis of the Na I D2 line of CARMENES (left) and HARPS-N data analysis (right).
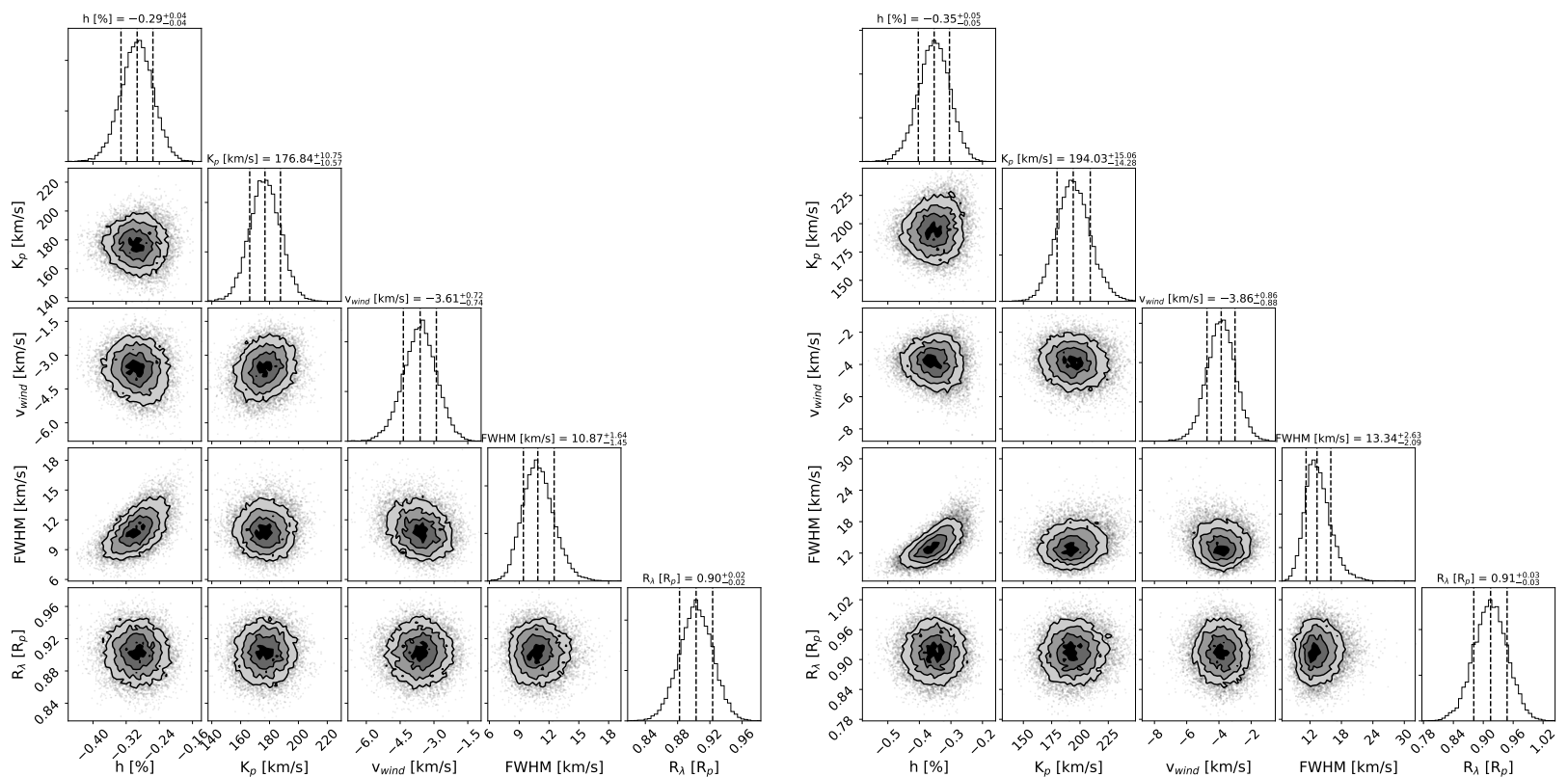

Fig. 7. Correlation diagrams of MCMC analysis of the Na I D1 line of CARMENES (left) and HARPS-N data analysis (right). 
A\&A 640, C6 (2020)
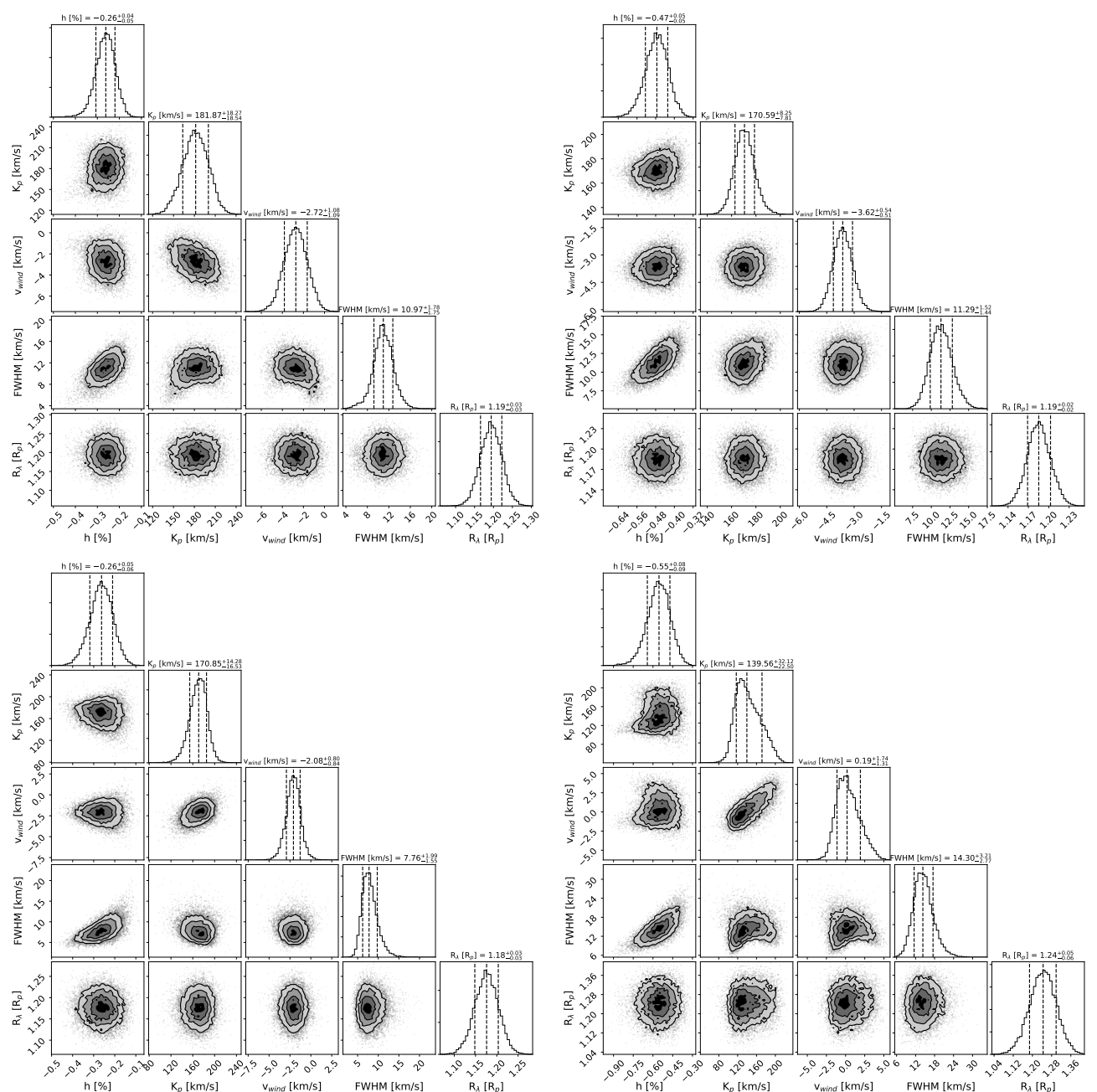

Fig. 8. Correlation diagrams of MCMC analysis of the Fe II triplet lines. The Fe II $\lambda 5018 \AA$ diagram is shown in the top left and Fe II $\lambda 5169 \AA$ is in the top right, both of which result from the HARPS-N combined data analysis. The Fe II $\lambda 5316 \AA$ diagrams are shown in the bottom panels: the HARPS-N analysis is on the left and the CARMENES analysis is on the right.

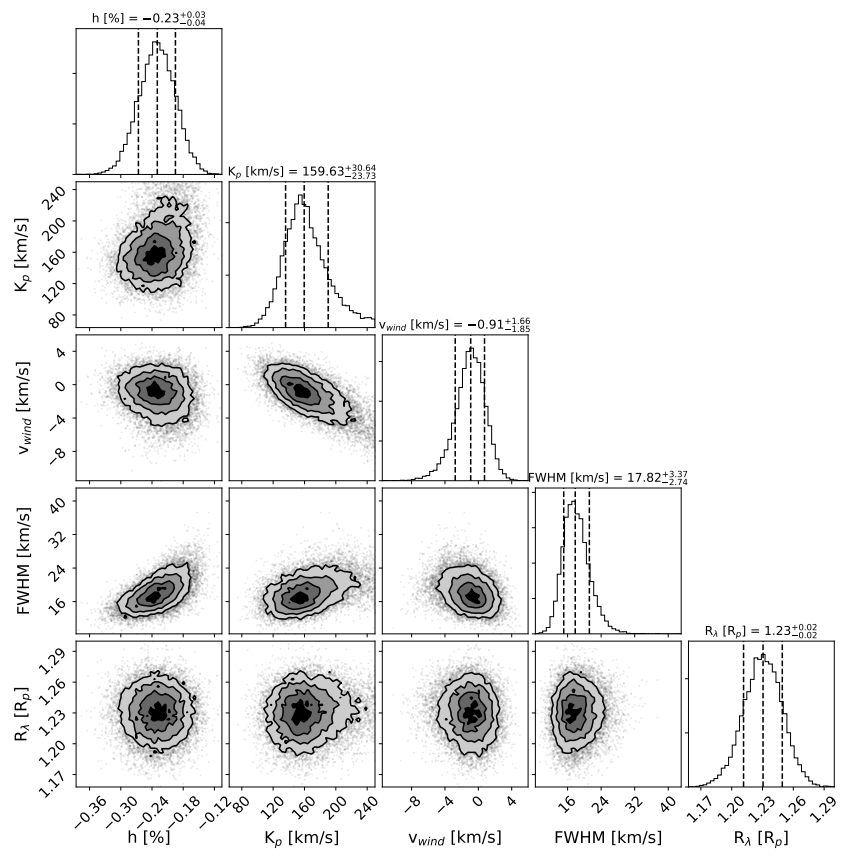

Fig. 9. Correlation diagrams of MCMC analysis of the Mg I line at $5173 \AA$ A. 